\title{
Erklärung zu den Beschränkungen der Verteidigung im Strafverfahren
}

In großer Sorge appellieren die unterzeichneten Hochschullehrer des Strafrechts an Bundestag, Bundesregierung und Bundesrat, mit der Beschneidung der rechtsstaatlich gebotenen Verteidigung im Strafverfahren einzuhalten.

$\mathrm{Zu}$ den wesentlichen Elementen der freiheitlich demokratischen Grundordnung gehört das Recht eines jeden Beschuldigten auf ein faires Verfahren, das die Gefahr von Fehlurteilen soweit menschenmöglich ausschaitet und die Uberlegenheit des staatlichen Strafverfolgungsapparats gegenüber dem beschuldigten Bürger durch eine starke Rechtsposition der Verteidigung auszugleichen sucht.

Die zu Beginn dieses Jahres in Kraft getretenen Gesetze haben - zugleich mit einer Machterweiterung der Staatsanwaltschaft, der richterliche Befugnisse übertragen worden sind - in schwerwiegender Weise in das Recht der Verteidigung eingegriffen. Die Voraussetzungen für Beschränkungen sind außerordentlich weit gefaßt und stehen zu der Schwere des Eingriffs in keinem Verhältnis. Verteidiger können aufgrund von Beschuldigungen ausgeschlossen werden, ohne daß diese in einer für eine Verurteilung ausreichenden Weise bewiesen zu werden brauchen. Das Recht des Verteidigers, in der Hauptverhandlung jederzeit Erklärungen abzugeben, ist beschnitten. Angeklagte können - bei bewußt herbeigeführter Verhandlungsunfähigkeit - in Abwesenheit und damit ohne wirkliche Verteidigungsmöglichkeit zu schwerer Strafe verurteilt werden.

In diesen Bestimmungen werden die Interessen der Strafverfolgung dem Schutz des beschuldigten Bürgers vor Fehlurteilen übergeordnet. Sie sind aus einer von Emotionen beladenen Atmosphäre geboren, auf schwebende Verfahren zugeschnitten und in einem Schnellverfahren verabschiedet worden, das eine politische und wissenschaftliche Auseinandersetzung über das Für und Wider von vornherein ausschloß.

Die Einwände gegen Inhalt und Zustandekommen gelten in noch höherem Maße gegen die ad-hoc-Gesetze, die sich jetzt im Gesetzgebungsverfahren befinden. Eine Uberwachung des Verkehrs zwischen Verteidiger und verhafterem Beschuldigten bis in das Stadium des Hauptverfahrens macht eine wirksame Verteidigung unmöglich. Ein Handel der Strafverfolgungsorgane mit dem "Kronzeugen", der sich Nachsicht gegenüber seinen Verbrechen durch belastende Aussagen gegen andere erkaufen kann, beschwört Falschaussagen und damit Verurteilungen auch Unschuldiger herauf.

Am Strafprozeßrecht eines Staates läßt es sich ablesen, wie ernst er die Sicherung des Bürgers gegen Fehlgriffe der Staatsgewalt nimmt. Die Bundesregierung hat in der Begründung zum Ersten Strafverfahrensreformgesetz erklärt, die Strafprozeßordnung müsse den Wertentscheidungen des Grundgesetzes über die Stellung des Einzelnen zur Gemeinschaft angepaßt werden. Für Reformen im Strafprozeßrecht, die auf dieses Ziel gerichtet sind, werden wir uns einsetzen. Die gegenwärtigen Rechtsänderungen aber weisen in die entgegengesetzte, die falsche Richtung.

Prof. Dr. Günter Bemmann (Universität Augsburg), Prof. Dr. Anne-Eva Brauneck (Universitär Gießen), Prof. Dr. Rolf-Peter Calliess (Technische Universitär Hannover), Priv. Doz. Dr. Friedrich Dencker (Universitär Bonn), Prof. Dr. Dr. Herberg Fiedler (Universität Bonn), Univ. Doz. Dr. Martin Fincke (Universität Regensburg), Prof. Dr. Wolfgang Frisch (Universität Bonn), Prof. Dr. Heinz Giehring 
(Universität Hamburg), Prof. Dr. Gerald Grünwald (Universitär Bonn), Prof. Dr. Winfried Hassemer (Universität Frankfurt), Prof. Dr. Joachim Herrmann (Universität Augsburg), Prof. Dr. Günther Jakobs (Universität Kiel), Prof. Dr. Herbert Jäger (Universität Frankfurt), Ass. Prof. Dr. Heike Jung (Universität Saarbrücken), Prof. Dr. Dr. h. c. Arthur Kaufmann (Universität München), Prof. Dr. Günther Kohlmann (Universität Köln), Prof. Dr. Detlef Krauß (Universität Saarbrücken), Prof. Dr. Justus Krümpelmann (Universität Mainz), Prof. Dr. Ernst-Joachim Lampe (Universitär Bielefeld), Prof. Dr. Klaus Lüderssen (Universität Frankfurt), Prof. Dr. Helmut Marquardt (Universität Bonn), Prof. Dr. Heinz Müller-Dietz (Universität Saarbrücken), Prof. Dr. Klaus Rolinski (Universität Regensburg), Prof. Dr. Hans-Joachim Rudolphi (Universität Bonn), Prof. Dr. Hinrich Rüping (Universität Bonn), Prof. Dr. Erich Samson (Universitär Kiel), Prof. Dr. Hans-Ludwig Schreiber (Universirät Göttingen), Priv. Doz. Dr. Bernd Schünemann (Universität München), Prof. Dr. Günter Stratenwerth (Universität Basel), Prof. Dr. Heinz Wagner (Universität Kiel), Prof. Dr. Jürgen Welp (Universität Münster).

\section{Todesurteile in Spanien?}

Seit 36 Jahren verfolgt das Franco-Regime seine Gegner mit willkürlicher Verhaftung, Folter und Mord. Die spanische Rechtsprechung in politischen Verfahren ist nichts anderes als eine makabre Farce. In Spanien kann die Exekutive verhaften und verurteilen. In Spanien kann man wegen eines Delikts zweimal verurteilt werden. In Spanien verstößt die Wahrnehmung grundlegender Menschenrechte gegen das Gesetz. In Spanien werden Anwälte wegen Beleidigung des Gerichts bestraft, wenn sie bei einer Verhandlung von Folterungen an ihren Mandanten berichten. In Spanien werden Anwälte, die in politischen Prozessen ein Mandat haben, systematisch eingeschüchtert, willkürlich verhaftet wie jene 26 Anwälte kürzlich in Madrid. In Spanien wurde in diesen Tagen von einem Militärgericht gegen die beiden Basken Juan Antonio Garmendia und Angel Otaegui die Todesstrafe beantragt! Garmendia wird beschuldigt, einen Guardia Civil erschossen, Otaegui Garmendia versteckt zu haben. Bei Auseinandersetzungen während ihrer Verhaftung wurde Otaegui schwer verletzt, erhielt Garmendia einen Schuß in den Kopf. Er ist nicht verhandlungsfähig. Man hat ihn 4 Monate (!) lang nach einer Operation von allen Kontakten mit der Außenwelt isoliert. In dieser Zeit soll er das Geständnis abgelegt haben, auf das sich die Anklage nun hauptsächlich stützt. Das Hauptverfahren beginnt in den nächsten Tagen. Ein genauer Termin ist selbst Germendias Anwalt noch nicht bekannt. In Spanien stehen zwei weitere Prozesse kurz bevor, bei denen Anträge auf Todesstrafe zu befürchten sind! Genoveva Forest, eine Ärztin, und die Volksschullehrerin Maria Luz, als Oppositionelle gegen das Regime bekannt, werden beschuidigt, am Bombenattentat auf ein Madrider Café beteiligt gewesen zu sein. Sie werden vor ein Militärgericht gestellt. Der Anschlag war möglicherweise die Tat rechtsradikaler Kreise. Das bisherige Verfahren gegen die beiden Frauen spottet jeder Beschreibung. Beide wurden gefoltert. Maria Luz wurde über 3 Monate (!) lang von jedem Kontakt zur Außenwelt isoliert. Die bisher von den Behörden vorgelegten Beweise bestehen im angeblichen Geständnis eines anderen Häftlings, der seinem Anwalt versichert hat, daß er nichts von einem Geständnis wisse. Genoveva Forest wird außerdem beschuldigt, zusammen mit dem Arbeiter Antonio Duran am Attentat auf den damaligen Ministerpräsidenten Carrero Blanco im Winter 1973 beteiligt gewe- 